Communications in Physics, Vol.25, No. 1 (2015), pp. 75-90

DOI:10.15625/0868-3166/25/1/5829

\title{
EVIDENCE FOR POLARIZED SOLAR FLARE EMISSION FROM SIMULTANEOUS RADIO OBSERVATIONS IN AUSTRALIA AND VIETNAM
}

\author{
NGUYEN THI THAO, NGUYEN THI PHUONG, PIERRE DARRIULAT, PHAM NGOC DIEP, \\ DO THI HOAI, PHAM TUYET NHUNG, AND PHAM TUAN ANH \\ VATLY, VNSC, VAST, 18 Hoang Quoc Viet, Nghia Do, Cau Giay, Hanoi, Vietnam
}

\begin{abstract}
A sample of 34 solar flares recorded in Hanoi using the VATLY radio telescope between April 2012 and January 2014 at frequencies of 1415 and $1417.6 \mathrm{MHz}$ is compared with the corresponding observations made at 1415 $\mathrm{MHz}$ by the solar observatory of Learmonth (Australia). While good overall agreement is obtained, a few differences are noted and studied in some detail. In particular, the use of different feeds in Hanoi and Learmonth reveals a case of very large polarization of the flare emission.
\end{abstract}

Keywords: radio astronomy, radio emission of the Sun, solar flares, polarization.

\section{INTRODUCTION}

As a by-product of observations [1,2] of the Sun using the VATLY radio telescope $[3,4]$, a sample of 34 solar flares [5] observed simultaneously in Hanoi and Learmonth [6] has been collected. These observations provide interesting material for a study of the perfomance of the VATLY radio telescope under conditions of important and rapid changes of the signal with time. The present work is an analysis of this data sample made in the spirit of revealing possible dysfunctions of the instrument which earlier studies would have been unable to reveal. The data sample under study includes 7 flares recorded in a first campaign of observation at $1415 \mathrm{MHz}$, between April and September 2012, and 27 flares recorded in a second campaign at $1417.6 \mathrm{MHz}$ between October 2013 and January 2014.

Hanoi and Learmonth are located at nearby longitudes $\left(105.8^{\circ} \mathrm{E}\right.$ and $114.1^{\circ} \mathrm{E}$ respectively) and at nearly opposite latitudes $\left(21.0^{\circ} \mathrm{N}\right.$ and $22.2^{\circ} \mathrm{S}$ respectively). The technical characteristics of the Learmonth radio telescope (Fig. 1) are essentially identical to those of the Hanoi telescope, apart from the use of a dipole rather than helical feed, implying detection of the average flux density rather than that of the right-handed component of the incident wave. The Learmonth observatory is staffed seven days a week from sunrise to sunset and contributes data to the US Air Force Weather Agency, to the US National Oceanic and Atmospheric Administration and to the Global Oscillation Network Group. Its long experience with solar measurements and its commitment to serve a large community make it a highly reliable source of data. The noise level is a factor $\sim 1.7$ lower for the Learmonth radio telescope than for that in Hanoi.

Details about the VATLY radio telescope and its performance have been published earlier $[3,4]$ and do not need to be repeated here. It is sufficient to recall that it is equipped with a fully steerable parabolic dish, $2.6 \mathrm{~m}$ in diameter, remotely adjustable in elevation and azimuth.

C2015 Vietnam Academy of Science and Technology 
It is operated at frequencies between 1400 and $1440 \mathrm{MHz}$. The reflected power is collected at focus by a left-handed helical feed, where it is locally pre-amplified, shifted to lower frequency using standard super-heterodyne, amplified and digitized. Standard data collection consists in a sequence of successive measurements at $\sim 8.2 \mathrm{~s}$ intervals, digitized in the form of a frequency histogram covering $\sim 1.2 \mathrm{MHz}$ in 156 bins of $\sim 7.8 \mathrm{kHz}$ each. The range is obtained by stitching together three sub-bandwidths. The telescope orientation is remotely adjustable. The angular aperture of the main lobe (the "beam") is well described by a Gaussian having a $\sigma$ of $2.3^{\circ}$ and the pointing accuracy is measured to be typically $0.22^{\circ}$ in $a \times \cos (h)$ and $0.11^{\circ}$ in $h$ where $a$ and $h$ are the azimuth and elevation respectively. An antenna efficiency factor of $\sim 65 \%$ has been measured, meaning a conversion factor of $1.25 \pm 0.09 \mathrm{~K} / \mathrm{kJy}$. The system temperature, including all sources of non astronomical origin, is of the order of $220 \mathrm{~K}$.
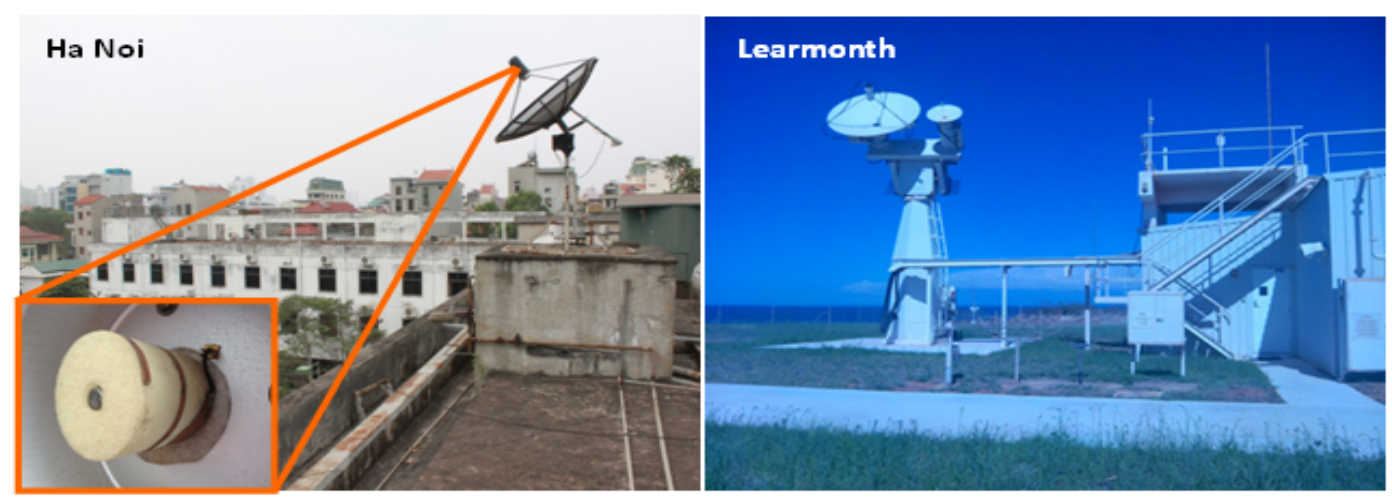

Fig. 1. The Hanoi (left) and Learmonth (right) radio telescopes. The former is on the roof of a small Hanoi building in an urban environment, the latter in an airport near the ocean. The insert shows the left-handed feed of the Hanoi telescope. A $1 \mathrm{~m}$ diameter antenna is also visible on the Learmonth picture.

\section{DATA REDUCTION}

The time dependence of the measured solar flux emitted during eruption of a large flare is illustrated in Fig. 2 that displays both the Hanoi and Learmonth observations. Each measurement is averaged over the $1 \mathrm{MHz}$ bandwidth. Averaging is done for us in the Learmonth case, the data being available on Internet [6] at $1 \mathrm{~s}$ intervals in solar flux units ( $\left.1 \mathrm{SFU}=10^{4} \mathrm{Jy}\right)$. In the Hanoi case, the measured antenna temperature is averaged over the 138 channels of a same frequency spectrum after having cleaned the data for possible radio frequency interferences (RFIs). The Hanoi measurements are taken at intervals of $\sim 8.2 \mathrm{~s}$ instead of $1 \mathrm{~s}$ in Learmonth. They are of the antenna temperature and are converted to SFU with a conversion factor of $12.5 \mathrm{~K} / \mathrm{SFU}$. They are used to generate a list of values available at each second by linear interpolation between successive measurements. This procedure implies that the VATLY radio telescope is blind to fine structures at the $1 \mathrm{~s}$ scale as illustrated in Fig. 3. In both Hanoi and Learmonth data some measurements are occasionally missing or faulty and replaced by interpolated values. The synchronism between the 
times at which flares are seen to occur, measured by a delay $\Delta t$ between the Learmonth and Hanoi measurements has a mean value of $1.8 \mathrm{~s}$ and an rms dispersion of $\pm 3.9 \mathrm{~s}$ in good agreement with expectation.

In most cases, the measurements of the quiet Sun flux made in Hanoi and Learmonth agree with each other: their ratio (Hanoi to Learmonth) has a mean value of 0.98 and an rms dispersion of 0.07 . In what follows, the Hanoi data are normalized to the Learmonth data in an interval of a few minutes preceding the flare.
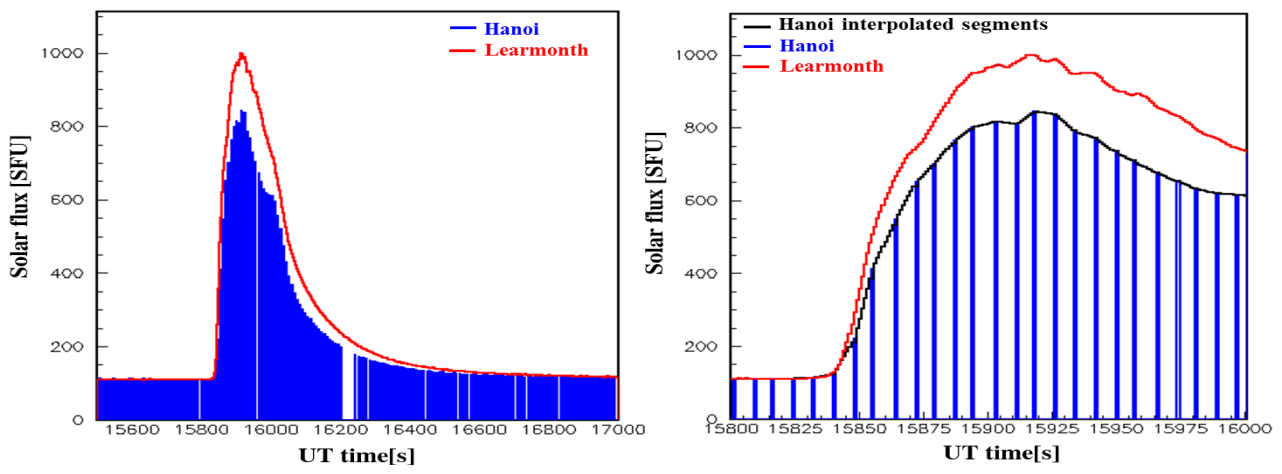

Fig. 2. Left: A large flare as seen in Learmonth (red) and in Hanoi (raw data, blue). The Hanoi data are converted to SFU using a conversion factor of $1.15 \mathrm{~K} / \mathrm{kJy}$ in order to have a same quiet Sun flux density as in Learmonth. The abscissa is UT time in seconds. In the Learmonth case, there is in principle one measurement each second. In the Hanoi case, there is, in principle, one measurement every $8.2 \mathrm{~s}$ or so. Right: A zoom on the start of the flare, displaying in addition the interpolated Hanoi data (black).

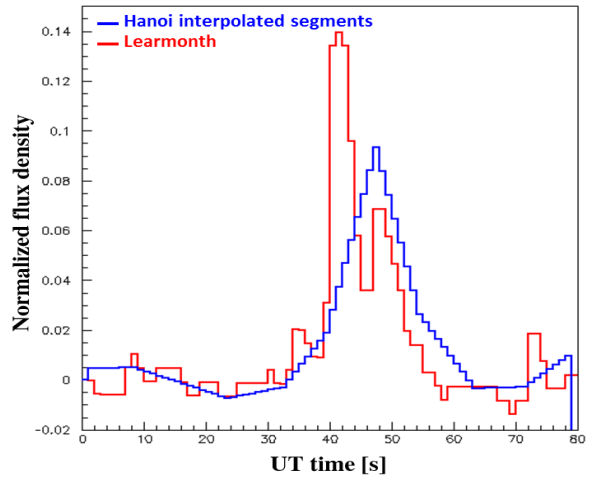

Fig. 3. Time dependence of Flare 18 displaying fine structure as detected by Learmonth (red) to which Hanoi (blue) is blind. The quiet Sun level has been subtracted and the flux densities normalized to the flare area. Segments associated with the interpolation performed between successive Hanoi measurements are clearly visible. 


\section{DISTURBED FREQUENCY SPECTRA}

In a very few cases disagreement between the Learmonth and Hanoi data has been observed, suggesting an anomalous functioning of the latter. We now address this issue.

Each frequency spectrum is fitted to a straight line. The quality of the fit is measured by a $\chi^{2}$ evaluated for a constant relative uncertainty of $2 \%$ and normalized to the number of degrees of freedom. Its distribution is displayed in Fig. 4 (left). We arbitrarily define as "bad fits" those having $\log _{10}\left(\chi^{2}\right)>0.1\left(\chi^{2}>1.26\right)$. The slope of the straight line, measured in ppm per $\mathrm{kHz}$, deviates by a quantity $\delta$ from its average value (slightly dependent on the central frequency, see [4]); its distribution is displayed in Figure 4 (center) for "good" and "bad" fits respectively. Having noticed that "bad fits" are often associated with slightly different amplitudes in each of the three sub-bandwidths, we define, for each frequency spectrum, an asymmetry $\alpha=\left\langle T_{\text {central }}\right\rangle$ $/<T\rangle_{\text {edges }}-1$ as the deviation from unity of the ratio between the mean antenna temperature measured in the central sub-bandwidth, $\langle T\rangle_{\text {central }}$, to the mean antenna temperature measured in the edge sub-bandwidths, $<T>_{\text {edges }}$. Its distribution is displayed in Fig. 4 (right) for "good" and "bad" fits respectively. As the data from the three sub-bandwidths, integrated over equal intervals of time, are read in sequence rather than simultaneously, it is not surprising that they display differences when the signal varies significantly during the measurement. If the signal increases or decreases uniformly during the measurement, the value of $\delta$ will be affected but those of $\alpha$ and $\chi^{2}$ will not. On the contrary, if the signal goes through or approaches an extremum during the measurement, the value of $\chi^{2}$ will be affected but those of $\alpha$ and $\delta$ will be less.
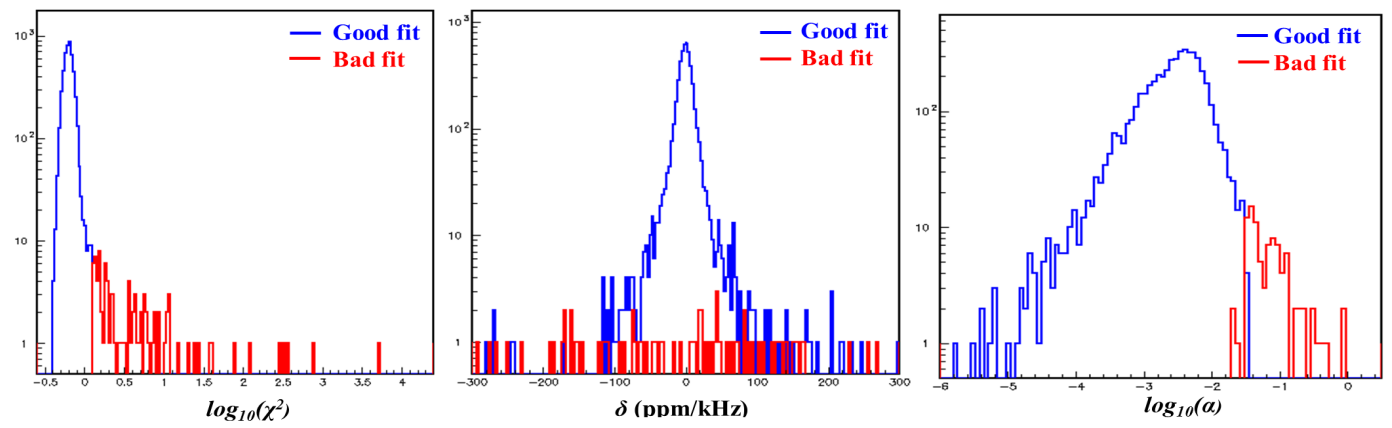

Fig. 4. Distributions of $\log _{10}\left(\chi^{2}\right)$ (left), of $\delta$ (in ppm/kHz, middle) and $\log _{10}(\alpha)$ (right). "Good" and "bad" fit values are shown in blue and red respectively. Log scales are used for the ordinates.

The distributions displayed in Fig. 4 show that the system is usually well behaved. In particular, the slope of the frequency spectrum is mostly Gaussian distributed with an rms of only $9 \mathrm{ppm} / \mathrm{kHz}$. While the "bad" fit values of $\delta$ span a broad range, those of $\alpha$ are confined to large values. Indeed, all spectra having $\log _{10}\left(\chi^{2}\right)<0.1\left(\chi^{2}<1.26\right)$ have $\log _{10}(\alpha)<-1.4(\alpha<0.04)$. This strong correlation between $\alpha$ and $\chi^{2}$ is confirmed in Fig. 5 where the two quantities are plotted together for the 94 spectra (out of 4995) having $\log _{10}\left(\chi^{2}\right)>0.1$. It implies that the main cause of deterioration of the quality of the fit is related with different flux densities recorded in each of the sub-bandwidth, and therefore a consequence of rapid changes of the signal level. 


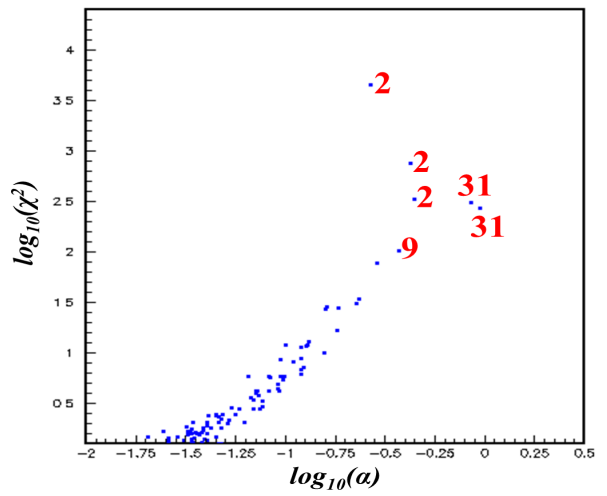

Fig. 5. Two-dimensional plot of $\log _{10}\left(\chi^{2}\right)$ vs $\log _{10}(\alpha)$ for "bad" fits. Flares having the larger values of $\chi^{2}$ are labelled as in Fig. 6.
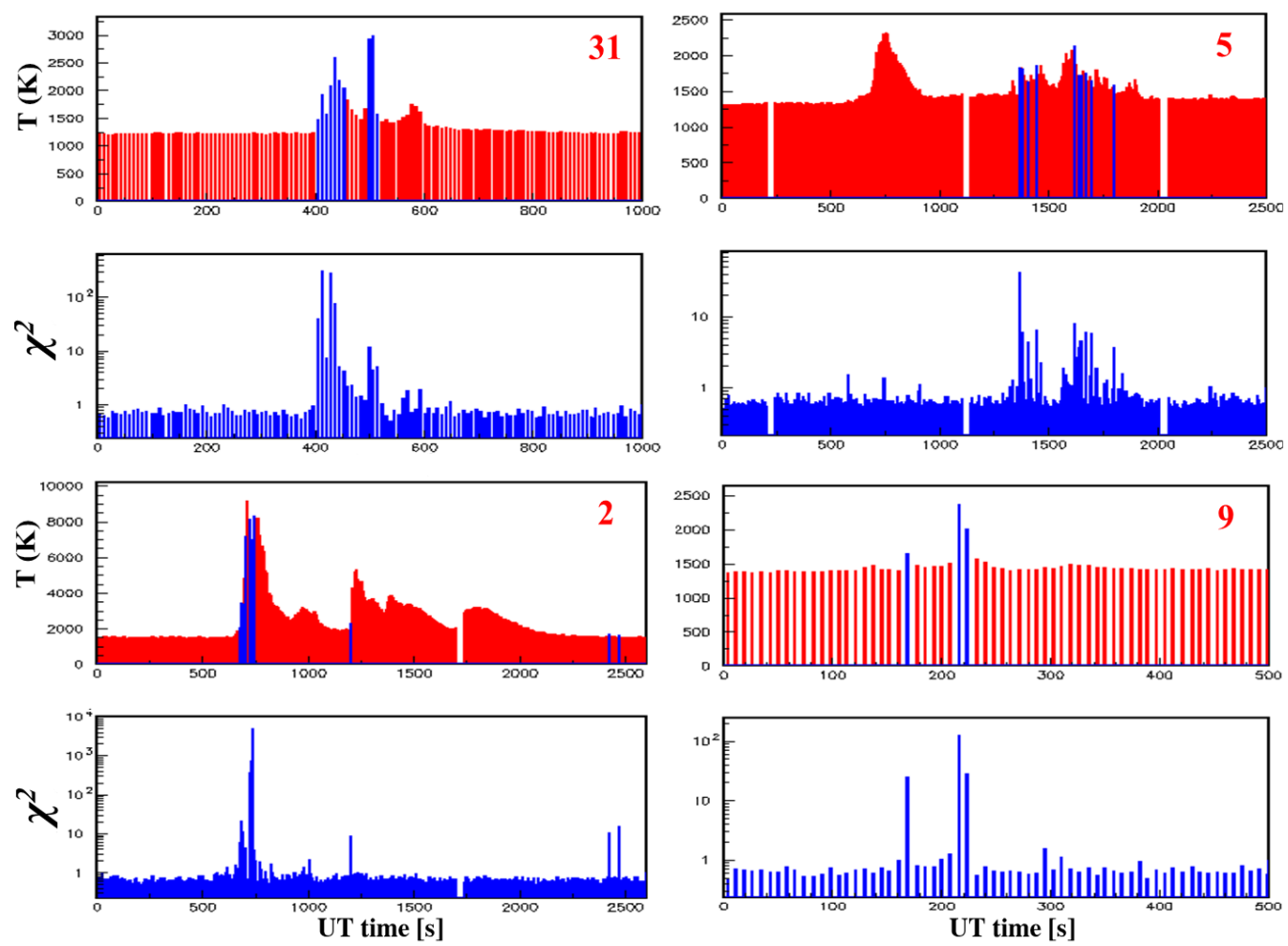

Fig. 6. The four flares having frequency spectra with the larger values of $\chi^{2}$. Each flare is illustrated by two panels, the top one displaying the variation of the antenna temperature vs time (in seconds) and the lower one displaying the variation of $\chi^{2}$. In the upper panels, measurements having $\chi^{2}>\sqrt{10}$ are shown in blue. 

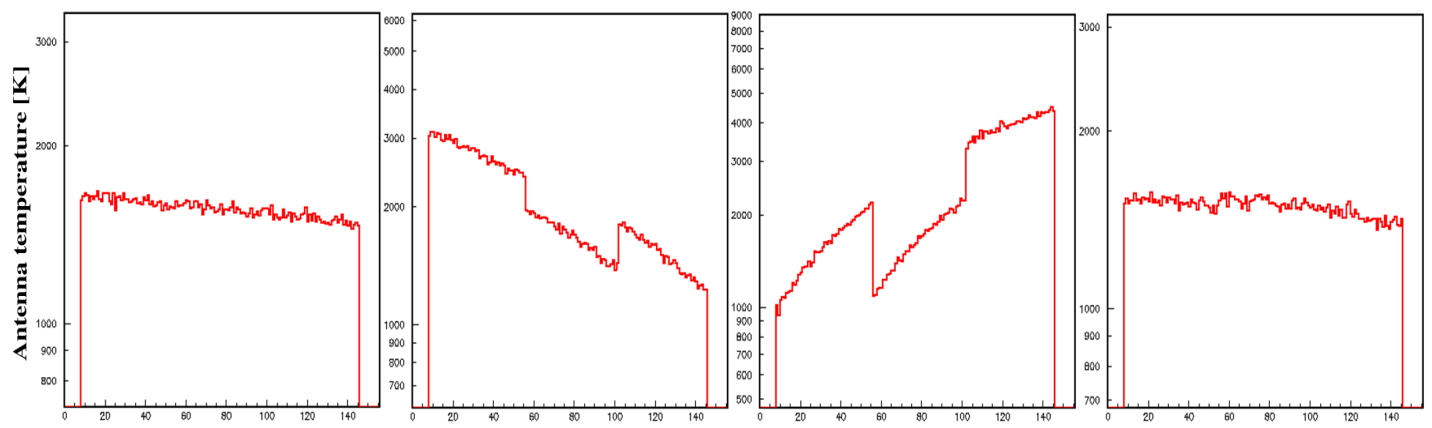

Fig. 7. Frequency spectra of a sequence of four successive measurements, the two central being "bad" fits (taken from Flare 5). The abscissa covers 1.2 MHz centered on 1417.6 $\mathrm{MHz}$
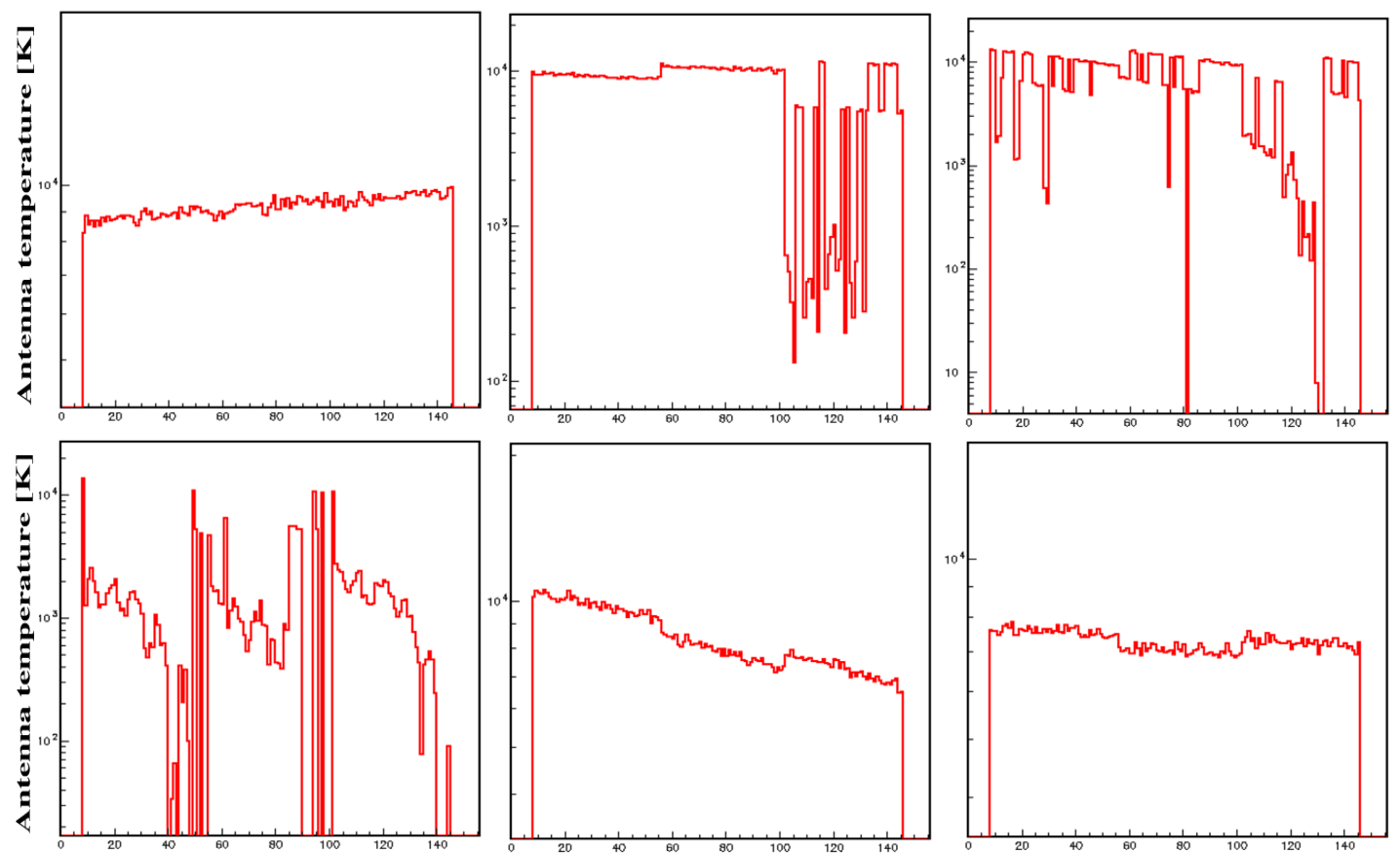

Fig. 8. Frequency spectra of the only sequence of measurements displaying dysfunctions of another type than simple fluctuations of $\delta$ and $\alpha$. They are taken from Flare 2 data. The abscissa covers $1.2 \mathrm{MHz}$ centered on $1417.6 \mathrm{MHz}$

In most "bad" fit cases, the dissymmetry between the central and the edge sub-bandwidths, which is responsible for the large value of $\chi^{2}$, is rather modest. A value of $\alpha$ equal to 0.2 $\left(\log _{10}(\alpha)=-0.7\right)$ typically means an error of only $\sim 7 \%$ on the flux density. We inspected by eye all spectra having $\log _{10}\left(\chi^{2}\right)>0.5$ in order to unveil possible dysfunctions of a different 
nature. This resulted in a sample of 45 measurements (out of a total of 4995) associated with 9 flares. Fig. 6 displays the four profiles including the spectra having the larger values of $\chi^{2}$. As expected, the steepness of the variation of the detected flux is a more important parameter than the flare amplitude in producing "bad" fits. A sequence of frequency spectra, displaying particularly strong $\delta$ and $\alpha$ variation, is displayed in Fig. 7. It illustrates well how "bad" fits are normally associated with important dissymmetry between the three sub-bandwidths. Of the 45 spectra inspected by eye, only three display severe dysfunctions going beyond a simple sub-bandwidth asymmetry. They are associated with the largest flare, labelled 2 in Figs. 5 and 6, and are displayed in Fig. 8.

In conclusion, in the vast majority of measurements, the instrument is well behaved and the evaluation of the antenna temperature is reliable to a precision of a few percent. In a few cases where the signal varies rapidly, the frequency spectrum has a slope that differs significantly from average and/or displays significant differences between the level of the central sub-bandwidth and that of the edge sub-bandwidths. However, this affects only modestly the flux density measurement. Only in the extreme case of three measurements of the largest Flare (\#2) spectra showing severe dysfunction (Fig. 8) have been excluded from the subsequent analysis.

\section{COMPARISON BETWEEN HANOI AND LEARMONTH OBSERVATIONS}

We now compare the flare data collected in Hanoi with those collected in Learmonth. In spite of the strong similarity between the profiles obtained by the two observatories, significant differences are present, which ask for an explanation. As a working hypothesis, we take the Learmonth observations as reference and accept that the Hanoi measurements may suffer instrumental problems, but we assume that perfect instruments should observe identical profiles. This is only true in the absence of polarization. While the Learmonth telescope will generally catch half of the power collected by the dish, the Hanoi telescope may catch it all or catch nothing depending on the handedness of the wave. A possible influence of the ionosphere, which is in quite different states in Hanoi and at Learmonth, is supposed to be negligible at our frequency.

In order to reveal possible differences between Learmonth and Hanoi observations, we evaluate the ratio $\rho=S_{H N} / S_{L M}$ of the Hanoi to Learmonth flux densities as a function of time and look for significant deviations from unity. We observe that important deviations are usually associated with large flares and with flares displaying rapid variations. We define for each flare the maximal value $\chi_{\text {max }}^{2}$ taken by $\chi^{2}$, the maximal deviation of $\rho$ from unity, $|1-\rho|_{\max }$ and the maximal value $S_{\max }$ of the flux density measured in Learmonth. Distributions of these parameters are displayed in Fig. 9.

Eight flares are seen to have $|1-\rho|_{\max }$ in excess of $20 \%$. Of these, three (Flares 2, 14 and 30) are the stronger flares in the sample. Fig. 10 (left) displays the distribution of $\log _{10}\left(S_{H N}\right)$ vs $\log _{10}\left(S_{L M}\right)$ for all flares. Flares 3, 30 and 2 deviate significantly from the mean. While Flares 2 and 30 are among the stronger, Flare 14, which is equally strong, displays no significant deviation, excluding effects caused exclusively by the amplitude of the signal, such as saturation of the electronics.

Small deviations between the Hanoi and Learmonth observations could be due to small differences in gains and/or system temperatures. In general, without knowing the gains and system temperatures of the two instruments, we may write $S_{L M}=\xi S_{H N}-\mu$ where $\xi-1$ and $\mu$ measure possible differences in gain and system temperature. As the two flux densities were normalized before the flare to a common quiet Sun value $S_{0}, \xi$ and $\mu$ must obey the relation $\mu=(\xi-1) S_{0}$ 
if a good fit is to be obtained before the flare. For each profile, we can find the values of $\xi-1$ and $\mu$ that give the best fit between the two sets of data. If they deviate only slightly from zero, the accuracy with which the system temperature and the gain of the Hanoi telescope have been evaluated can be blamed. However, some of the values taken by $\xi$ and $\mu$, displayed in Fig. 10 right, are too large for such an explanation. While showing the expected correlation and a nucleus of concentration around $\xi \sim 1.2$ and $\mu \sim 20$, as expected for a Hanoi system temperature of $\sim 220$ $\mathrm{K}$ and a quiet Sun level of $\sim 120 \mathrm{SFU}$, they reveal the existence of significant deviations. Particularly spectacular is the case of Flare 3, a small flare that followed Flare 2 one hour later, which stands out far away from the other flares.
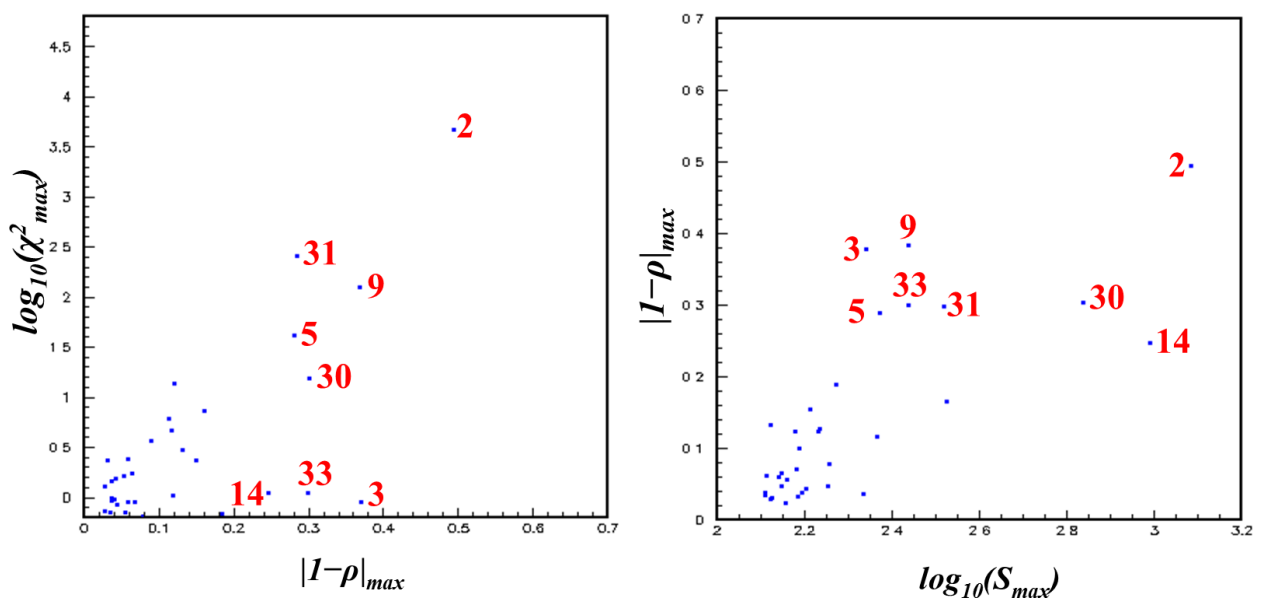

Fig. 9. Left: distribution of $\log _{10}\left(\chi_{\max }^{2}\right)$ vs $|1-\rho|_{\max }$. Right: distribution of $|1-\rho|_{\max }$ vs $\log _{10}\left(S_{\max }\right)$.
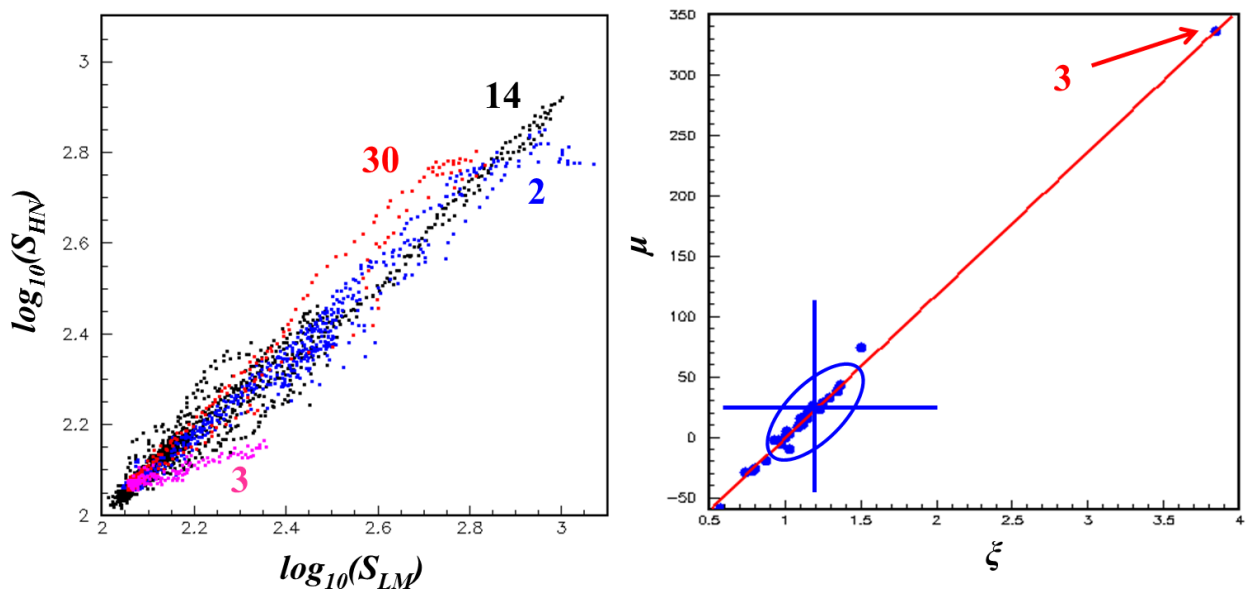

Fig. 10. Left: Distribution of $\log _{10}\left(S_{H N}\right)$ vs $\log _{10}\left(S_{L M}\right)$. Right: Distribution of $\mu$ vs $\xi$. The line is for $S_{0}=119 \mathrm{SFU}$. The cross indicates the expected average values. The ellipse indicates the set of measurements used to evaluate the quantity $\delta \xi$ in Sec. V. 


\section{INTERPRETATION IN TERMS OF POLARIZED FLARE EMISSION}

The analyses of the preceding sections have not revealed any dysfunction of the Hanoi telescope and have given confidence in the reliability of its observations, an invitation to consider seriously the possibility that the differences between the Hanoi and Learmonth observations are due, at least in part, to the polarization of the flare emission.

Indeed, important polarizations are common in the radio emission of solar flares and are routinely recorded in solar observatories such as Nobeyama in Japan [7]. Models of the relevant mechanisms are available in the literature [8].

In order to compare flare emissions rather than total emissions, we define for each flare a profile $F$ obtained by subtracting the quiet Sun signal from the total detected signal. In order to reduce errors resulting from a possible time shift between the Learmonth and Ha Noi data, we use time bins of $10 \mathrm{~s}$ instead of $1 \mathrm{~s}$. The quiet Sun signal is obtained by linear interpolation between the signals measured before and after the flares. We checked that using instead a constant quiet Sun signal, as measured only before the flare, would not significantly affect the results because the quiet Sun levels before and after a flare are very close to each other.

We obtain the polarization $P$ of the flare emission from the ratio between the flux densities of the flare emissions measured in Hanoi and Learmonth,

$F_{H N} / F_{L M}=2 R /(R+L)=1+(R-L) /(R+L)=1+P$ where, $R$ and $L$ stand for the right-handed and left-handed flux densities respectively.

Subtracting the quiet Sun signal from the total signal to obtain the flare signal implies that the system temperature drops out from the evaluation of the flare emission. However, when we normalize the pre-flare quiet Sun flux measured in Hanoi to that measured in Learmonth to ease the comparison between the two sets of data, this operation depends slightly on the system temperature assumed for the Hanoi telescope, which we take equal to $215 \pm 30 \mathrm{~K}$. The uncertainty of $\pm 30 \mathrm{~K}$ translates in a first uncertainty on the value of $P$. A second uncertainty results from a possible time shift between the Hanoi and Learmonth measurements, which we take as $\pm 4 \mathrm{~s}$. Both uncertainties may be very small in the case where the polarization is small. In particular, they essentially cancel in the quiet Sun regime. In order to account for possible inaccurate evaluations of the system temperature and gain of the Hanoi telescope with respect to those of the Learmonth telescope, we introduce a third uncertainty evaluated from the concentration of events displayed in Fig. 10 (right). Assuming that their dispersion, $\Delta \xi \sim 9 \%$, is purely instrumental, namely that the associated flares are unpolarized, we find that the uncertainty $\delta \xi$ on $\xi$ translates in a same uncertainty $\delta P$ on $P$. We take as global uncertainty $\Delta P$ the quadratic sum between the three contributions. To the extent that part of the two former contributions contributes to the third, this is a conservative evaluation of the global uncertainty. It is also conservative to the extent that part of the value of $\Delta \xi$ is likely to be due to polarization (which is not expected to exactly cancel).

Another cause of error is a possible imperfect design of the Hanoi feed. Indeed, while its circumference is equal to the wavelength as it should, its pitch is only $3 \mathrm{~cm}$ instead of the quarter wavelength $(5.3 \mathrm{~cm})$ expected. There exists an abundant literature on helical antennas [9], but we were unable to find a reference commenting on the purity of the handedness of the detected wave. Assuming that the Hanoi telescope detects a fraction $\varepsilon>0$ of the left-handed component of the incident wave, the polarization $P^{\prime}$ measured as $F_{H N} / F_{L M}-1$ reads $P^{\prime}=(1-2 \varepsilon) P$. To the extent that $\varepsilon<<1$, a reasonable assumption, the effect is simply to lower the amplitude of the 
polarization by a constant factor.

Finally, we remark that the use of $10 \mathrm{~s}$ wide time bins makes the analysis blind to any fluctuation of the polarization on a significantly smaller time scale. In fact, earlier studies of solar flares with time resolutions several orders of magnitude smaller than the coarse bin size used here have revealed the existence of such fluctuations. However, whatever polarization is measured in the present work, it is averaged over time and the result is therefore lower than the maximal polarization possibly reached on a smaller time scale. Therefore, the observation in the present study of a very large polarization in a particular instance is in no way spoiled by the use of a broad time resolution.

Flare 33, a C3.9 flare peaking at 140 SFU above quiet Sun, displays an apparent time mismatch of $\sim 10 \mathrm{~s}$ between Learmonth and Hanoi.Such mismatch may occasionally happen [Owen Giersch, private communication] and makes Flare 33 unsuitable for a reliable polarization measurement. We retain for further scrutiny any other flare either having an unpolarized flux density $\left(F_{L M}\right)$ in excess of $100 \mathrm{SFU}$ or displaying large significant values of $P$. Precisely, we define as reliable polarization measurements those having both $\Delta P<15 \%$ and $F_{L M}>25$ SFU. Two M flares, Flares 31 and 9, display too rapid flux density variations to obey these constraints. This leaves Flares 30, 2, 3, 5, 14, 15 and 21 for further scrutiny. In these cases, the studies presented in the preceding sections have made it evident that none of the possible instrumental effects that have been considered could produce a significant artificial polarization.

Table 1. Flare properties.

\begin{tabular}{|c|c|c|c|c|c|c|c|}
\hline Label & $\begin{array}{c}\text { Date } \\
(\mathrm{ymd})\end{array}$ & $\begin{array}{c}\text { Starting } \\
(\mathrm{UT})\end{array}$ & Class & $\begin{array}{c}\operatorname{Max}\left(F_{L M}\right) \\
{[\mathrm{kSFU}]}\end{array}$ & $\begin{array}{c}\operatorname{Max}(P) \\
{[\%]}\end{array}$ & Nobeyama & Active region \\
\hline 30 & 120703 & $3: 36$ & C9.9 & $\sim 0.45$ & $\sim+30$ & Yes & 1515 \\
\hline 2 & 131025 & $7: 53$ & $\mathrm{X} 1.7$ & $\sim 0.90$ & $\sim-30$ & No & 1882 \\
\hline 3 & 131025 & $9: 01$ & - & $\sim 0.10$ & $\sim-70$ & No & 1882 \\
\hline 5 & 131028 & $4: 41$ & M5.1 & $\sim 0.09$ & $\sim \pm 10$ & Yes & 1875 \\
\hline 14 & 131108 & $4: 20$ & X1.1 & $\sim 0.85$ & $\sim-20$ & Yes & 1890 \\
\hline 15 & 131110 & $5: 08$ & X1.1 & $\sim 0.18$ & $\sim 0$ & Yes & 1890 \\
\hline 21 & 131207 & $7: 17$ & M1.2 & $\sim 0.08$ & $\sim 0$ & No & 1909 \\
\hline
\end{tabular}

The main parameters [10] of the retained flares are listed in Table 1 and their profiles are displayed in Fig. 11. The measured polarizations $P$ of the flare emission are displayed in Fig. 12. The pair of Flares $2+3$ is particularly spectacular. Flare 2 is an X1.7 flare that erupted from active region 1882, just after it had emerged from the eastern limb of the Sun (Fig. 13 left). It displays a rich time structure. It was followed seven hours later by an X2.9 flare erupting from the same Sun spot, too late however to have been caught by our and Learmonth telescopes. Flare 3 erupted about one hour after Flare 2 and reaches very large $P$ values. Measurements made at Learmonth at other frequencies (Fig. 13 right) show that it is not seen at frequencies in excess of $\sim 2 \mathrm{GHz}$ while the brightness of Flare 2 keeps slowly rising with frequency, giving evidence for their very different natures. 

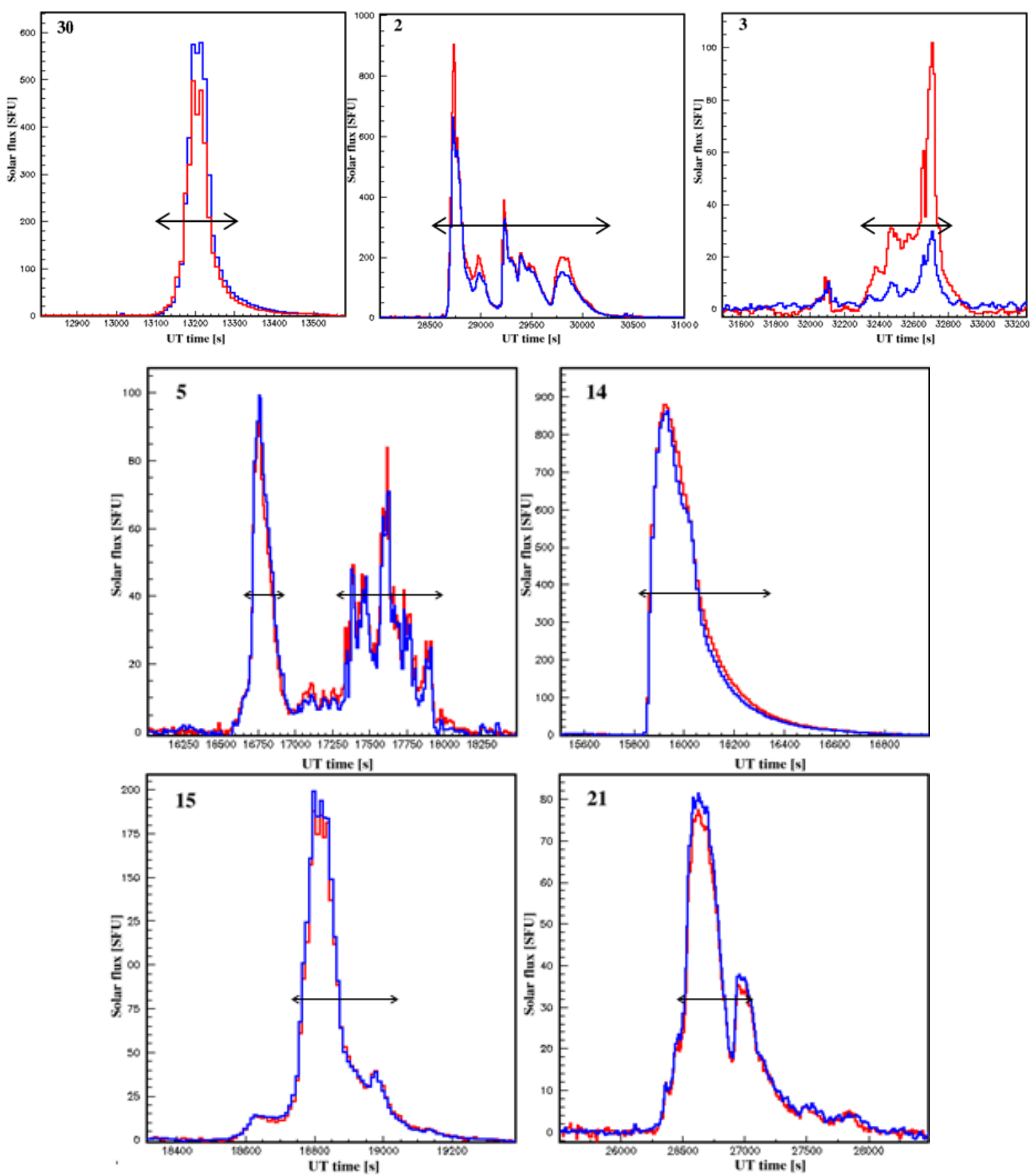

Fig. 11. Flare profiles as measured in Learmonth (red) and Hanoi (blue). The arrows indicate the intervals over which polarization is displayed in Fig. 12 as being reliably measured.

In both Learmonth and Hanoi, the quiet Sun antenna temperature returns to its original level between Fares 2 and 3 and after Flare 3. Flare 3 stands out (Fig. 10 left) as being in particularly 

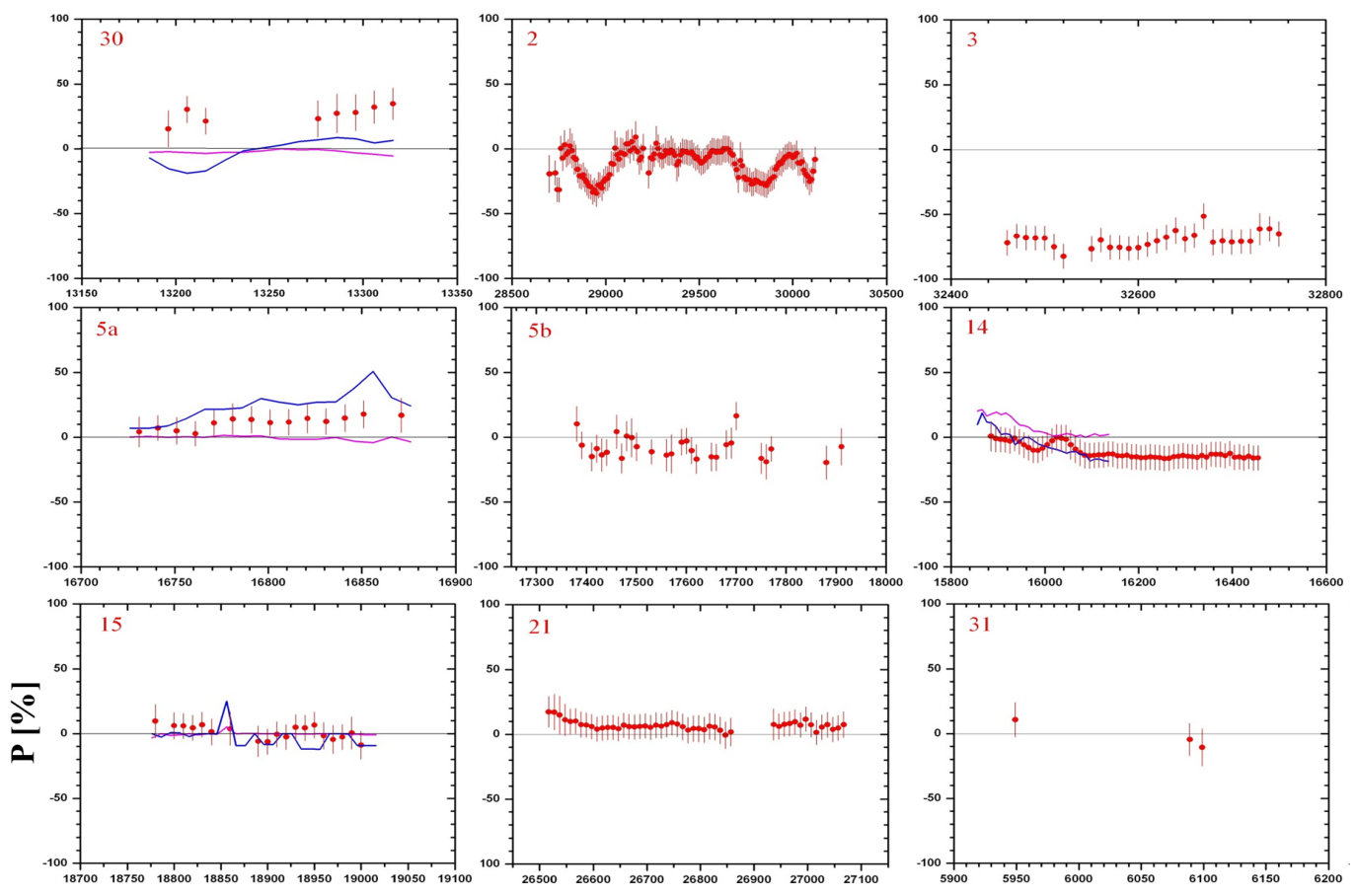

UT time [s]

Fig. 12. Polarizations measured for the flares listed in Table 1 (red) over the time intervals where reliable measurements are available (as indicated in Fig. 11). Nobeyama polarizations (blue) measured at $1 \mathrm{GHz}$ (magenta) and $2 \mathrm{GHz}$ (blue) are shown when available. Also shown is the polarization of Flare 31, a M2.9 flare peaking at 280 SFU, which erupted from active region 1515 on July 6th, 2012 and was measured unpolarized in Nobeyama.

strong disagreement between the two observatories, implying that at least one of the two telescopes responds differently to emission from the flare and emission from the quiet Sun. Another way to illustrate the puzzling situation of Flare 3 is from its position in the $\mu$ vs $\xi$ plot of Fig. 10 right, showing that no sensible values of the gain and system temperature can be invoked to explain the effect.

Fig. 14 compares the flux densities of Flares $2+3$ measured in Learmonth and in the solar observatory of San Vito dei Normanni [11], in southern Italy. This observatory operates radio telescopes essentially identical to those at Learmonth. The Learmonth and San Vito measurements, when simultaneously available, are in good agreement. This is likely to exclude possible effects of the Hanbury Brown-Twiss type [12] causing important differences between the two observatories on one hand and Hanoi on the other.

Major radio bursts, of the Tenflare type [13], meaning more than twice quiet Sun at 10 $\mathrm{cm}$ wavelength, were active during the periods covered by Flares 2, 3 and 14. While ionospheric activity is expected to be much stronger in Hanoi than in Learmonth and San Vito because of its 

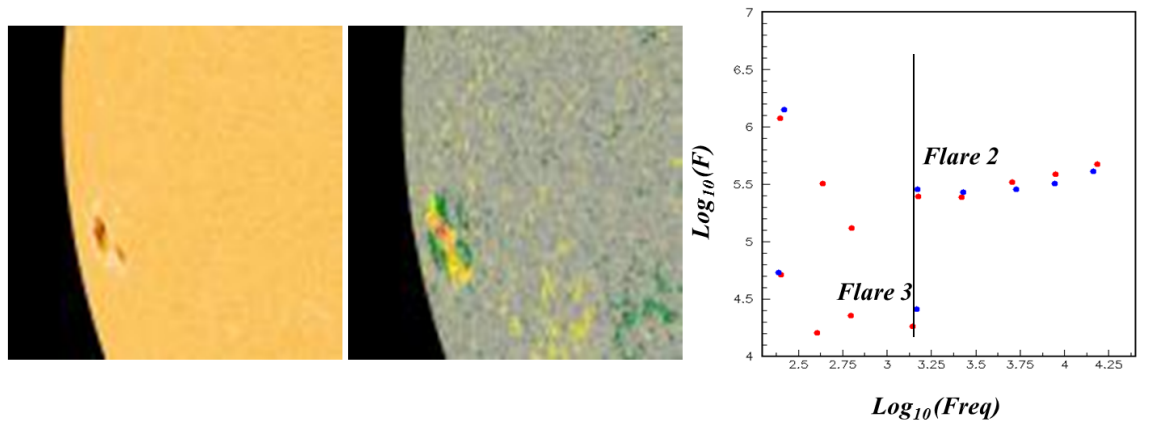

Fig. 13. Left: Optical map and magnetogram of Sun spot 1882 from where Flares 2 and 3 erupted. Right: Distribution of the decimal logarithm of the integrated flux densities (SFUs) measured in Learmonth for Flares 2 and 3 at Learmonth (red) and San Vito (blue) as a function of the decimal logarithm of the frequency $(\mathrm{MHz})$. Flare 3 is undetected beyond $2 \mathrm{GHz}$. The line is at $1.42 \mathrm{GHz}$.
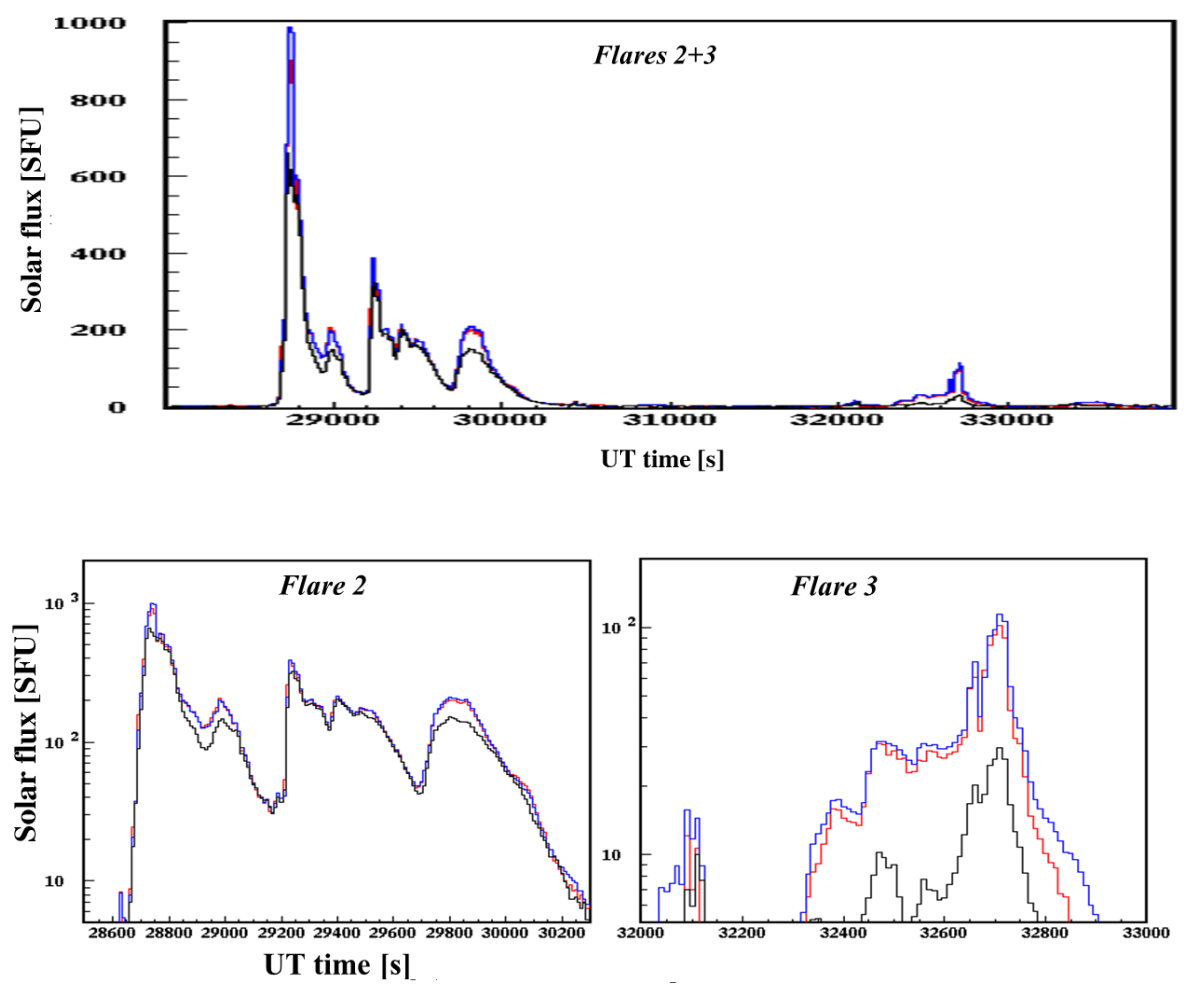

Fig. 14. Comparison between the flux densities (SFU) measured in Learmonth (red), San Vito (blue) and Hanoi (black) for the pair of Flares 2+3. In many cases the Learmonth and San Vito values are indistinguishable.

exceptionally high S4 scintillation index [14], this should not affect differently the quiet Sun and flare contributions. 
NGUYEN THI THAO et al.

Delayed decimetric bursts displaying a very high degree of circular polarization have been previously observed and examples of unusually strong such events are described in the literature [15].

Flare 14 is an X1.1 flare, accompanied, as was Flare 2, by important Coronal Mass Ejection, a type II/IV sweep frequency event and a Tenflare radio burst. However, it is single-peaked at strong variance with Flare 2.

Flare 30, contrary to Flares 2 and 14, is weak in X-ray emission. Flares 5, 15 and 21 are weaker and are consistent with being unpolarized.

Four of the flares listed in Table 1, Flares 30, 5, 14 and 15, have been detected by the Nobeyama solar polarimeters. Unfortunately, the Sun had set in Japan when the pair of Flares 2+3 erupted. The Nobeyama polarimeters operate at 1 and $2 \mathrm{GHz}$, providing two frequencies bracketing the Hanoi /Learmonth frequency. Fig. 12 compares the polarizations measured in Nobeyama with those obtained in the present work. The steep and irregular frequency dependence of the polarization of flare emissions, as observed in Nobeyama, makes a comparison difficult. Yet, the mismatch between Nobeyama and Ha Noi/Learmonth data on Flare 30 is a cause of concern as none of the effects that have been considered until now can be blamed for such poor agreement.

\section{SUMMARY}

We have studied 34 solar flares that have been observed simultaneously in Hanoi and Learmonth; we have compared their profiles as measured in the two observatories with the aim of revealing possible instrumental effects affecting the Hanoi telescope.

A first result is the observation of disturbances of the frequency spectra measured in Hanoi that are strongly enhanced by the presence of solar flares. Such disturbances had already been observed as associated with radio frequency interferences or transients on the line [4]. They are well understood and consist in inequalities of the fluxes detected in each of the three sub-bandwidths that are stitched together to produce the total bandwidth and are associated with rapid variations of the detected signal. In most cases, they only have a small incidence on the accuracy of the measurement of the antenna temperature. In a very few cases, however, they cause the system to fail completely (three such measurements were found in the sample under study and have been illustrated in Fig. 8).

A detailed study has revealed the presence, in several cases, of significant differences between the flux densities measured in Hanoi and Learmonth. Some of these have been identified as being the result of the different time resolutions of the two telescopes. A few others have been interpreted as giving evidence for significant polarization of the flare emission. However, comparison with measurements made at Nobeyama failed to give strong support to this interpretation. Particularly spectacular is the case of a small flare, peaking at $\sim 100$ SFU above quiet Sun and following an X1.7 flare after a delay of $\sim 1$ hour, which displays a polarization of $\sim 70 \%$. In this case, the fact that the quiet Sun fluxes measured before, in between and after the pair of flares are in very good agreement between Hanoi and Learmonth makes it difficult to invoke another effect than a strong polarization of the flare emission. 


\section{ACKNOWLEDGMENTS}

We are indebted to the Learmonth Solar Observatory staff, who are making their data available to the public. We are deeply grateful to Dr Owen Giersch and Dr Alan Rogers for having kindly and patiently answered many of our questions and made us benefit of their experience by making several very useful suggestions and comments. N.H.P. Thanh and B.V. Tuan, master students at the University of Sciences and Technology of Hanoi , who spent a two month internship in the laboratory, took part in the early phase of the analysis. We thank Dr Sujin Kim for help

in accessing Nobeyama data and Pr Kiyoto Shibasaki for having introduced us to the physics of decimetric flares. Financial support is acknowledged from the Institute for Nuclear Science and Technology (VINATOM/MOST), the Vietnam National Foundation for Science and Technology Development (NAFOSTED) under grant number 103.08-2012.34, the World Laboratory, the Odon Vallet Foundation and the Rencontres du Viet Nam.

\section{REFERENCES}

[1] N. V. Hiep et al., Sol. Phys. 289(3) (2014), 939-950.

N. T. Phuong (on behalf of VATLY) presented at 2nd South East Asia Young Astronomer Collaboration, November 19-22, Bandung, Indonesia, 2013.

P. T. Nhung (on behalf of VATLY) presented at the Conference Windows on the Universe, August 11-17, Quy Nhon, Vietnam, 2013.

[2] P. N. Diep et al., Correlated Oscillations Due to Similar Multipath Effects Seen in Two Widely Separated Radio Telescopes, Publications of the Astronomical Society of Australia, 31, e029 doi:10.1017/pasa.2014.24.

N. T. Phuong (on behalf of VATLY), presented at Workshop on Astronomy and Astrophysics in Vietnam, April 7-11, Institute for Nuclear Science and Technology, Hanoi , Viet Nam.

P. N. Diep (on behalf of VATLY), presented at 12th Asia-Pacific Region IAU Meeting, Daejeon, Korea, 2014.

N. T. Phuong, Solar and other observations using the VATLY radio telescope, Master thesis, in preparation.

[3] N. V. Hiep et al., Comm. Phys. 22 (2012) 365.

N. V. Hiep (on behalf of VATLY), presented at the Second Academic Conference on Natural Science for Master and $\mathrm{PhD}$ students from Cambodia, Laos, Malaysia and Vietnam, 11-15 October 2011, Vinh, Vietnam.

P. T. Anh (on behalf of VATLY) presented at the 7th Annual Conference of the Thai Physics Society, May 9-12, 2012, Phranakhon Si, Ayutthaya, Thailand.

N. V. Hiep (on behalf of VATLY) presented at the 1st South East Asian Young Astronomers Collaboration (SEAYAC) Meeting, November 5-9, 2012, Puerto Princesa City, Palawan, Philippines.

[4] N. T. Phuong et al., Comm. Phys. 24 (2014) 257-266. DOI:10.15625/0868-3166/24/3/3981

[5] B. V. Tuan, A study of the six major solar flares recently detected from Hanoi and Learmonth at $1415 \mathrm{MHz}$ compared with other frequencies, internship report presented at University of Science and Technology of Hanoi, Master Space and Applications, September, 2014.

N. H. P. Thanh, A study of 34 solar flares detected by Hanoi and Learmonth telescope at $1415 \mathrm{MHz}$ between 2012 and 2014, internship report presented at University of Science and Technology of Hanoi, Master Space and Applications, September, 2014.

[6] Australian Government, Bureau of Meteorology, Radio and Space Weather Services, http://www.ips.gov.au/World_Data_Centre/1/10.

[7] http://solar.nro.nao.ac.jp/north/html/event/

[8] See for example M. Karlicky and M. Barta, Nonlinear Processes in Geophysics (2004) 11: 471 and references therein.

[9] J. D. Kraus, A Helical-Beam Antenna Without a Ground Plane, IEEE Antennas and Propagation Magazine, April 1995 , p. 45.

J. D. Kraus, Antennas, McGraw-Hill, 1950.

J. D. Kraus and R. J. Marhefka, Antennas: for All Applications, third edition, McGraw-Hill, 2002. 
See also http://www.w1ghz.org/antbook/conf/Helical_feed_antennas.pdf and references therein for an excellent summary.

[10] http://www.nasa.gov/content/goddard/sun-emits-2nd-solar-flare-in-2-days/ TESIS http://www.tesis.lebedev.ru/en/about_tesis.html;

NOAA, http://www.swpc.noaa.gov/ftpmenu/warehouse/2013.htlm; http://solar.physics.montana.edu/max_millennium/data_archives.shtml.

[11] http://www.ngdc.noaa.gov/stp/space-weather/solar-data/solar-features/solar-radio/rstn-1-second/san-vito/2013/

[12] R.Hanbury Brown and R. Q. Twiss, Nature 178/4541 (1956) 1046.

[13] Space Weather Highlights, SWPC PRF 1991, 28 October 2013, http://www.swpc.noaa.gov/weekly/2013_WeeklyPDF/prf1991.pdf.

[14] IPS: 2012, Australia Government Bureau of Meteorology, Radio and Space Weather Services, http://www.ips.goc.au/Satellite/6/3.

[15] E. W. Cliver, S. M. White and K. S. Balasubramaniam, The Astrophysics Journal 743 (2011) 145 and references therein. 